\title{
SCIENCE OF HUMAN MEASURES WORKSHOP: SUMMARY AND CONCLUSIONS
}

\section{CONTENTS}

Page

OVERVIEW OF THE WORKSHOP ...............................................................................

SUMMARY OF PLENARY SESSION BRIEFINGS ……………………………………....

The Science of Human Measures: Anticipating and Meeting Army Needs ..............................2

U.S. Army Human Capital Strategy: Challenges for Human Measures .......................................5

Selecting, Developing, and Retaining Soldiers with Heart......................................................

Assessment Essential to Training Effectiveness and Readiness .................................................

How we Know what we Know about Soldiers' Attitudes and Behaviors .................................12

SUMMARY OF PANEL 1 DISCUSSION: ASSESSING ATTITUDES AND APTITUDES ....15

Assessing Aptitude ……………………………………………………………15

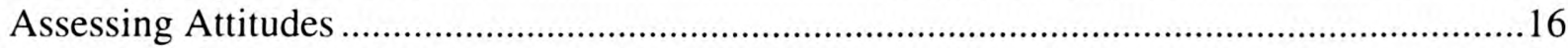

Panel Conclusions and Recommendations ..........................................................................17

SUMMARY OF PANEL 2 DISCUSSION: ASSESSING MENTAL AGILITY ………………....19

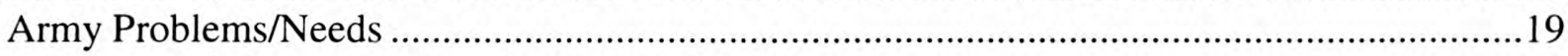

Adequacy of Current Approaches to Meet Army Needs ..........................................................20

New High Payoff Approaches ..............................................................................................2

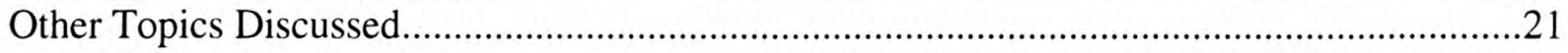

Panel Conclusions and Recommendations ………………………………………….....22

SUMMARY OF PANEL 3 DISCUSSION: ASSESSING INDIVIDUAL PERFORMANCE ....23

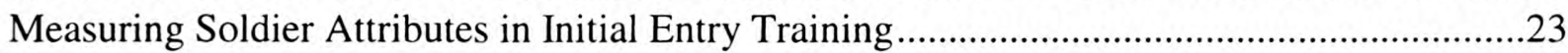

Evidence Centered Design .................................................................................................24

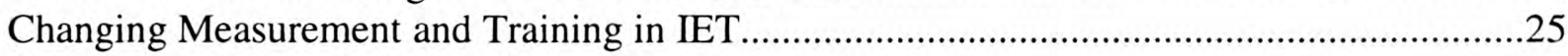

Developing Measures for Soldier Attributes.....................................................................26

Prototype Measures for Selected Soldier Attributes ……………………………………........27

Training Knowledge, Skills, and Abilities........................................................................29

Panel Conclusions and Recommendations .........................................................................30

SUMMARY OF PANEL 4 DISCUSSION: ASSESSING NEW TRAINING PROGRAMS .......32

Panel Leader Discussion Papers .....................................................................................

General Considerations .....................................................................................................

Training and Educational Programs ......................................................................................35

New System Training..........................................................................................................

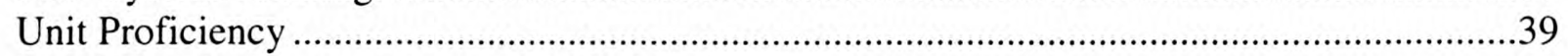

Panel Conclusions and Recommendations ...........................................................................4

METRICS WORKSHOP FINAL CONCLUSIONS …………………………………….....42

Measurement Feeds Assessment...................................................................................42

Good Measurement Can Not Be Developed from Poorly Defined Constructs .........................43

Good Measurement Includes More Than Just Questionnaires ....................................................43

Maintaining the Army's Human Measurement Capability .........................................................4 